\title{
Interval Based Finite Elements for Uncertainty Quantification in Engineering Mechanics
}

\author{
Rafi L. Muhanna ${ }^{1}$ and Robert L. Mullen ${ }^{2}$ \\ 1 School of Civil and Environmental Engineering \\ Georgia Institute of Technology \\ Atlanta, GA, USA \\ rafi.muhanna@gtsav.gatech.edu \\ 2 Department of Civil and Environmental Engineering \\ University of South Carolina \\ Columbia, SC, USA \\ rlm@sc.edu
}

\begin{abstract}
This paper illustrates how interval analysis can be used as a basis for generalized models of uncertainty. When epistemic uncertainty is presented as a range and the aleatory is based on available information, or when random variables are assigned an interval probability, the uncertainty will have a Probability Bound (PB) structure. When Interval Monte Carlo (IMC) is used to sample random variables, interval random values are generated. Interval Finite Element Method (FEM) is used to propagate intervals through the system and sharp interval solutions are obtained. Interval solutions are sorted and PBs of the system response are constructed. All relevant statistics are calculated characterizing both aleatory and epistemic uncertainty. The above mentioned sequence is presented in this work and illustrative examples are solved.
\end{abstract}

Keywords: interval, finite elements, reliability, aleatory, epistemic, probability bounds.

\section{Introduction}

The presence of uncertainty in all aspects of life is evident. However, quantifying uncertainty is always advancing. There are various ways in which types of uncertainty might be classified. One is to distinguish between "aleatory" (or stochastic) uncertainty and "epistemic" uncertainty. The first refers to underlying, intrinsic variability of physical quantities, and the latter refers to uncertainty which might be reduced with additional data or information, or better modeling and better parameter estimation [1].

Probability theory is the traditional approach to handling uncertainty. This approach requires sufficient statistical data to justify the assumed statistical distributions. Analysts agree that, given sufficient statistical data, probability theory describes the stochastic uncertainty well. However, probabilistic modeling cannot handle situations with incomplete or little information on which to

A. Dienstfrey and R.F. Boisvert (Eds.): WoCoUQ 2011, IFIP AICT 377, pp. 265-279, 2012.

(C) IFIP International Federation for Information Processing 2012 
evaluate a probability, or when that information is nonspecific, ambiguous, or conflicting [2], 3], and [4. Many generalized models of uncertainty have been developed to treat such situations, including fuzzy sets and possibility theory [5], Dempster-Shafer theory of evidence [6], 7], random sets [8, probability bounds [9], 2], and [10, imprecise probabilities [4], convex models [11], and others.

These generalized models of uncertainty have a variety of mathematical descriptions. However, they are all closely connected with interval analysis 12 . For example, the mathematical analysis associated with fuzzy set theory can be performed as interval analysis on different $\alpha$ levels [13] and [14]. Fuzzy arithmetic can be performed as interval arithmetic on $\alpha$ cuts. A Dempster-Shafer structure [6] and [7 with interval focal elements can be viewed as a set of intervals with probability mass assignments, where the computation is carried out using the interval focal sets. Probability bounds analysis [9], [2, and [10] is a combination of standard interval analysis and probability theory. Uncertain variables are decomposed into a list of pairs of the form (interval, probability). In this sense, interval arithmetic serves as the calculation tool for generalized models of uncertainty. A short description of probability bounds is given in the next section.

\section{Probability Bounds}

Probability Bounds (PB) identifies a specific mathematical framework for analysis when precise discrete probabilities (or PDF) are not completely known [4]. Probability bounds are normally associated with epistemic sources of uncertainty where the available knowledge is insufficient to construct precise probabilities. Calculations on PB can be conducted using Monte Carlo methods combined with an interval finite element method [15], or by using various discretization methods.

The arithmetic for piecewise constant discretization on PB can be found in publications such as [16], [17, [18, [10]. Let the behavior of the system be modeled by a function $y=f(x)$, where $x=\left(x_{1}, x_{2}, \ldots, x_{n}\right)$ is the parameter vector, and each $x_{i}$ is represented by a probability-bounds structure. The CDF of a particular value $y^{*}, F\left(y^{*}\right)$, is to be determined. The objective of probabilitybounds analysis is to obtain an interval to bound the precise probability $F\left(y^{*}\right)$ of interest (in the classical sense). The numerical implementation of probabilitybounds analysis can be done using interval analysis and the Cartesian product method. The general procedure can be found in publications such as [19], [20], 21, and 10.

For example, Fig. 1 depicts probability-bounds circumscribing a normal distribution whose mean is sure to lie within the interval $[5.6,6]$ and whose standard deviation is 1 . Such bounds can result from the addition of a normally distributed random variable with a mean of 5.6 and a standard deviation of 1 with a variable bounded between 0 and 0.4 .

Probability-bounds structure can also arise by forming probability distributions of intervals. In this context, probability-bounds structure is mathematically analogous to a standard discrete probability distribution except that the 


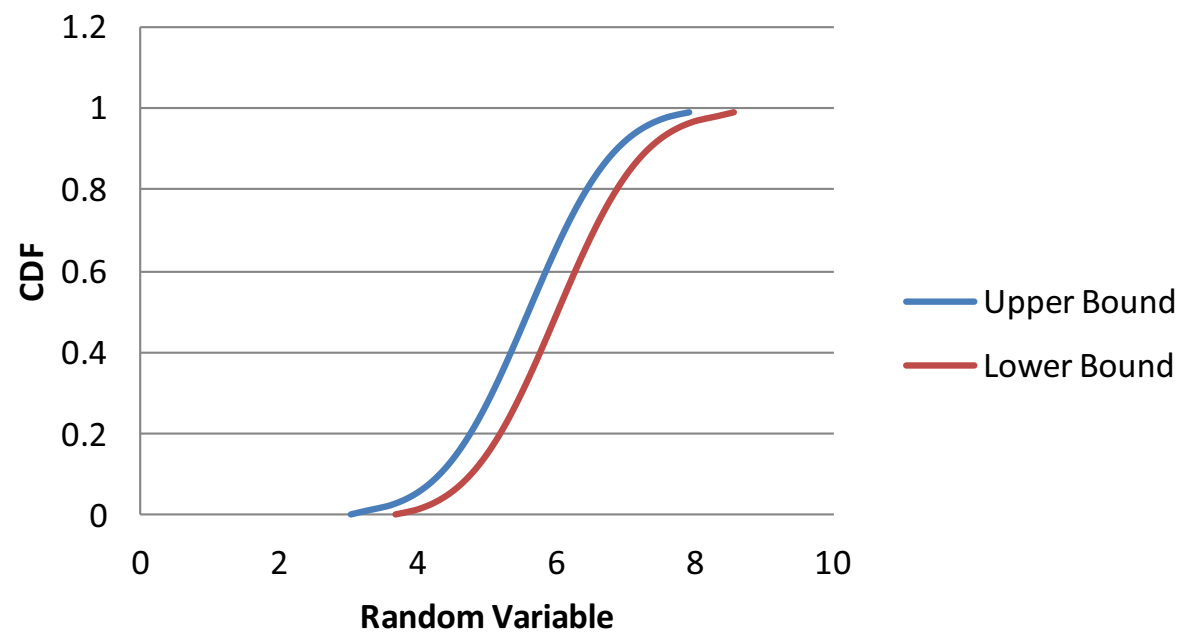

Fig. 1. Probability-bounds associated with normal distribution with mean $=[5.6,6]$ and standard deviation $=1$

probability mass is assigned to an interval rather than to a precise point; thus, the probability-bounds structure can be specified as a list of pairs of the form (interval, probability mass).

The advantage of the probability-bounds approach is that it can capture a wider range of uncertainties than the standard probabilistic approach. On the other hand, the standard probability distribution and interval number are two degenerate cases of probability-bounds structure; thus the probability-bounds approach provides a general framework for handling problems with a mixture of interval-based information and standard probabilistic information.

When and if additional knowledge of a system is obtained, the probability bounds can be refined. Just as interval and scalar calculations can be easily mixed, conventional precise probabilities and probability bounds may be mixed in a single calculation. However, how to accomplish this in a computationally efficient method for finite element analysis is unresolved. In this work interval Monte Carlo (IMC) will be used.

\section{Interval Monte Carlo}

When a random variable is described in a probability-bound structure, one natural approach for sampling such a random variable is the use of interval Monte Carlo (IMC). A discretization approach can be used as well [15]; however, this approach has not yet been developed for the general case. IMC has been proposed to generate fuzzy numbers in [22] and for the first time for structural reliability in [15].

The first step in the implementation of interval Monte Carlo simulation is the generation of intervals in accordance with the prescribed probability bounds. 


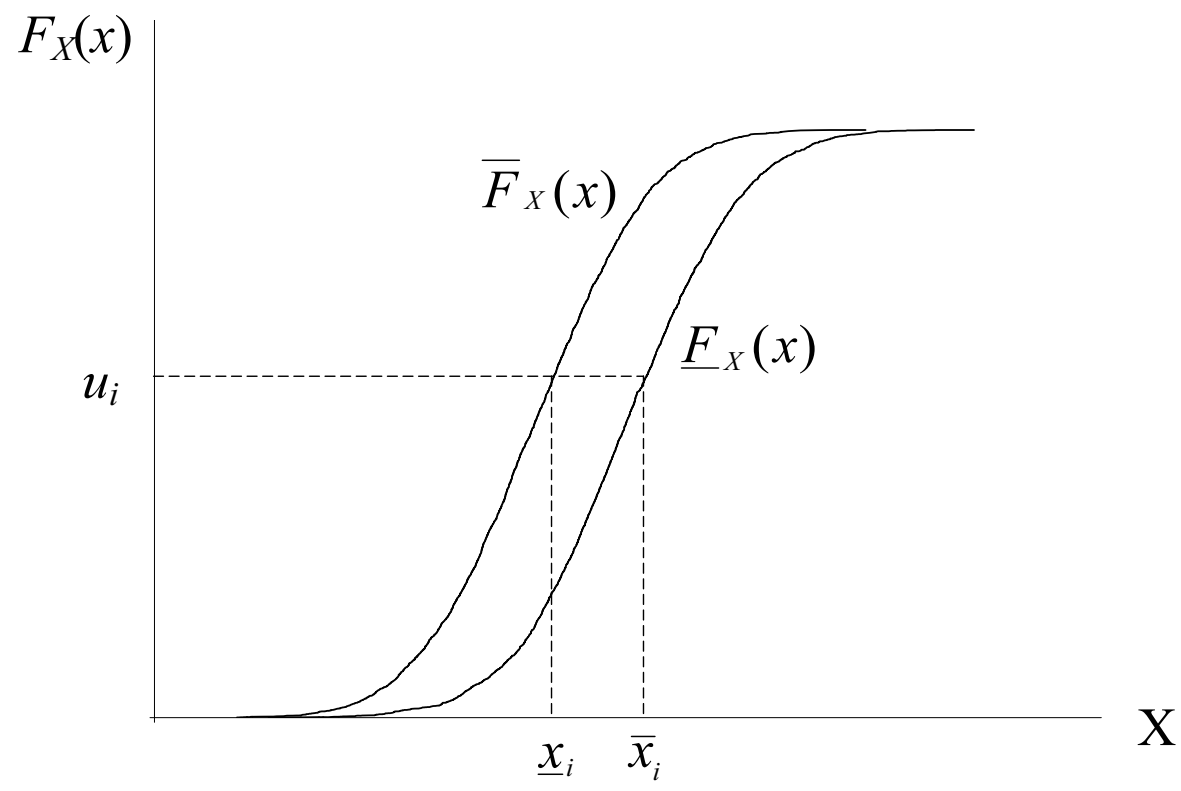

Fig. 2. Generation of random number from distribution with probability-bound structure

The inverse transform method is often used to generate random numbers. Consider a random variable $x$ with $\operatorname{CDF} F(x)$. If $\left(u_{1}, u_{2}, \ldots, u_{m}\right)$ is a set of values from the standard uniform variate, then the set of values

$$
x_{i}=F_{x}^{-1}\left(u_{i}\right), \quad i=1,2, \ldots, m
$$

will have the desired CDF $F(x)$. The inverse transform method can be extended to perform random sampling from a probability bound. Suppose that an imprecise CDF $F(x)$ is bounded by $\underline{F}(x)$ and $\bar{F}(x)$, as shown in Fig. 2, For each $u_{i}$ in Eq. (1), two random numbers are generated

$$
\underline{x}_{i}=\bar{F}_{x}^{-1}\left(u_{i}\right) \quad \text { and } \quad \bar{x}_{i}=\underline{F}_{x}^{-1}\left(u_{i}\right)
$$

Such a pair of $\underline{x}_{i}$ and $\bar{x}_{i}$ form an interval $\left[\underline{x}_{i}, \bar{x}_{i}\right]$ which contains all possible simulated numbers from the ensemble of distributions for a particular $u_{i}$. The method is graphically demonstrated in Fig. 2 for the one-dimensional case. The next step is to solve for the generated interval values using interval finite elements described in the next section.

\section{Interval Finite Element Methods}

One of the main features of interval arithmetic is its capability of providing guaranteed results. However, it has the disadvantage of overestimation if variables 
have multiple occurrences in the same expression. For example, if $x$ is an interval, evaluation of the function $f(x)=x-x$ using naive rules of interval arithmetic will not return zero but rather an interval that contains zero. Such situations lead to extremely pessimistic results, and have discouraged some researchers of pursuing further developments using interval representations.

The Finite Element Method (FEM) is a numerical method for solving differential and partial differential equations with enormous applications in different fields of the sciences and engineering. Interval Finite Element Methods (IFEM) have been developed for the last 15 years to handle the analysis of systems for uncertain parameters described as intervals. Since the early development of IFEM during the mid-1990s of the last century [23, 24, 25, 26, 27, and 28, researchers have focused, among other issues, on two major problems: the first is how to obtain solutions with reasonable bounds on the system response that make sense from a practical point of view, or in other words, with the least possible overestimation of their bounding intervals; the second is how to obtain reasonable bounds on the derived quantities that are functions of the system response.

The most successful approaches for overestimation reduction are those which relate the dependency of interval quantities to the physics of the problem being considered; details on these developments can be found in the works of the authors and their collaborators [24, [29], 30], and 31.

A brief description of IFEM formulation is presented below, details can be found in the authors' work [31. The two major issues in this formulation are:

1. Reducing overestimation in the bounds on the system response due to the coupling and transformation in the conventional FEM formulation as well as due to the nature of used interval linear solvers (Muhanna and Mullen, 2001).

2. Obtaining the secondary (derived) variables such as forces, stresses, and strains of the conventional displacement FEM along with the primary variables (displacements) and with the same accuracy of the primary ones. Previous interval methods calculate secondary variable from interval solutions of displacement which result in a significant overestimation.

\subsection{Discrete Structural Models}

In steady-state analysis, the variational formulation for a discrete structural model within the context of the Finite Element Method (FEM) is given in the following form of the total potential energy functional [32] and 33.

$$
\Pi=\frac{1}{2} U^{T} K U-U^{T} P
$$

with the conditions

$$
\frac{\partial \Pi}{\partial U_{i}}=0 \quad \text { for all } i
$$

where $\Pi, K, U$, and $P$ are total potential energy, stiffness matrix, displacement vector, and load vector respectively. For structural problems the formulation 
will include both direct and indirect approaches. For the direct approach the strain $\epsilon$ is selected as a secondary variable of interest, where a constraint can be introduced as $C_{2} U=\epsilon$. For the indirect approach constraints are introduced on displacements of the form $C_{1} U=V$ in such a way that Lagrange multipliers will be equal to the internal forces. $C_{1}$ and $C_{2}$ are matrices of orders $m \times n$ and $k \times n$, respectively, where $m$ is the number of displacements constraints, $k$ is the number of strains, and $n$ is the number of displacements degrees of freedom. We note that $V$ is a constant and $\epsilon$ is a function of $U$. We amend the right-hand side of Eq. (3) to obtain

$$
\Pi^{*}=\frac{1}{2} U^{T} K U-U^{T} P+\lambda_{1}^{T}\left(C_{1} U-V\right)+\lambda_{2}^{T}\left(C_{2} U-\epsilon\right)
$$

where $\lambda_{1}$ and $\lambda_{2}$ are vectors of Lagrange multipliers with dimensions $m$ and $k$, respectively. Invoking the stationarity of $\Pi^{*}$, that is $\delta \Pi^{*}=0$, we obtain

$$
\left(\begin{array}{cccc}
K & C_{1}^{T} & C_{2}^{T} & 0 \\
C_{1} & 0 & 0 & 0 \\
C_{2} & 0 & 0 & -I \\
0 & 0 & -I & 0
\end{array}\right)\left(\begin{array}{c}
U \\
\lambda_{1} \\
\lambda_{2} \\
\epsilon
\end{array}\right)=\left(\begin{array}{c}
P \\
V \\
0 \\
0
\end{array}\right)
$$

The solution of Eq. (6) will provide the values of the dependent variable $U$ and the derived ones $\lambda_{1}, \lambda_{2}$, and $\epsilon$ at the same time.

The present interval formulation, which will be introduced in the next section, is an extension of the Element-By-Element (EBE) finite element technique developed in the work of the authors [29].

The main sources of overestimation in the formulation of IFEM are the multiple occurrences of the same interval variable (dependency problem), the width of interval quantities, the problem size, and the problem complexity, in addition to the nature of the used interval solver of the interval linear system of equations. While the present formulation is valid for the FEM models in solid and structural mechanics problems, the truss model will be used here to illustrate the applicability and efficiency of the present formulation without any loss of generality.

The current formulation modifies the displacement constraints used in the previous EBE formulation to yield the element forces as Lagrange Multipliers directly and the system strains. All interval quantities will be denoted by nonitalic boldface font. Following the procedures given in [31] we obtain the interval linear system

$$
\left(\begin{array}{cccc}
\mathbf{K} & C_{1}^{T} & C_{2}^{T} & 0 \\
C_{1} & 0 & 0 & 0 \\
C_{2} & 0 & 0 & -I \\
0 & 0 & -I & 0
\end{array}\right)\left(\begin{array}{c}
\mathbf{U} \\
\boldsymbol{\lambda}_{1} \\
\boldsymbol{\lambda}_{2} \\
\mathbf{\epsilon}
\end{array}\right)=\left(\begin{array}{c}
\mathbf{P} \\
0 \\
0 \\
0
\end{array}\right)
$$

where $\mathbf{K}$ is an interval matrix of dimension (dof $\times$ dof), where dof is the sum of element degrees of freedom and the free node degrees of freedom. It consists of the individual elements' local stiffness and zeros at the bottom corresponding to the free nodes' degrees of freedom. 
The accuracy of the system solution depends mainly on the structure of Eq. (7) and on the nature of the used solver. The solution of the interval system (7) provides the enclosures of the values of dependent variables which are the interval displacements $\mathbf{U}$, interval element forces $\boldsymbol{\lambda}_{\mathbf{1}}$, the multiplier $\boldsymbol{\lambda}_{\mathbf{2}}$, and the element's interval strains $\boldsymbol{\epsilon}$. An iterative solver is discussed in the next section.

\subsection{Iterative Enclosures}

The best known method for obtaining very sharp enclosures of interval linear system of equations that have the structure introduced in Eq. (7) is the iterative method developed in the work of Neumaier and Pownuk 34. The current formulation results in the interval linear system of equations given in (7) which can be transformed to have the following general form:

$$
(K+B \mathbf{D} A) \mathbf{u}=a+F \mathbf{b}
$$

where $\mathbf{D}$ is diagonal. Furthermore, defining

$$
C:=\left(K+B D_{0} A\right)^{-1}
$$

where $D_{0}$ is chosen to ensure invertablility (often $D_{0}$ is selected as the midpoint of $\mathbf{D})$, the solution $\mathbf{u}$ can be written as

$$
\mathbf{u}=(C a)+(C F) \mathbf{b}+(C B) \mathbf{d} .
$$

To obtain a solution with tight interval enclosure we define two auxiliary interval quantities,

$$
\begin{aligned}
& \mathbf{v}=A \mathbf{u} \\
& \mathbf{d}=\left(D_{0}-\mathbf{D}\right) \mathbf{v}
\end{aligned}
$$

which, given an initial estimate for $\mathbf{u}$, we iterate as follows:

$$
\mathbf{v}=\{(A C a)+(A C F) \mathbf{b}+(A C B) \mathbf{d}\} \cap \mathbf{v}, \quad \mathbf{d}=\left\{\left(D_{0}-\mathbf{D}\right) \mathbf{v}\right\} \cap \mathbf{d}
$$

until the enclosures converge, from which the desired solution $\mathbf{u}$ can be straightforwardly obtained.

This formulation allows obtaining the interval displacement $\mathbf{U}$ and the accompanied interval derived quantities $\boldsymbol{\lambda}_{\mathbf{1}}, \boldsymbol{\lambda}_{\mathbf{2}}$, and $\boldsymbol{\epsilon}$ with the same accuracy. A number of examples are introduced in the next section.

\section{$5 \quad$ Examples}

Fig. 3. shows a planar truss. The reliability of this structure using single deflection criteria has been presented by Zhang, et al. 15. Two limit states are considered in this work, the serviceability and the strength. The deflection limit at the mid-span is set to $2.4 \mathrm{~cm}$, and the allowable stress in any member is set 200 


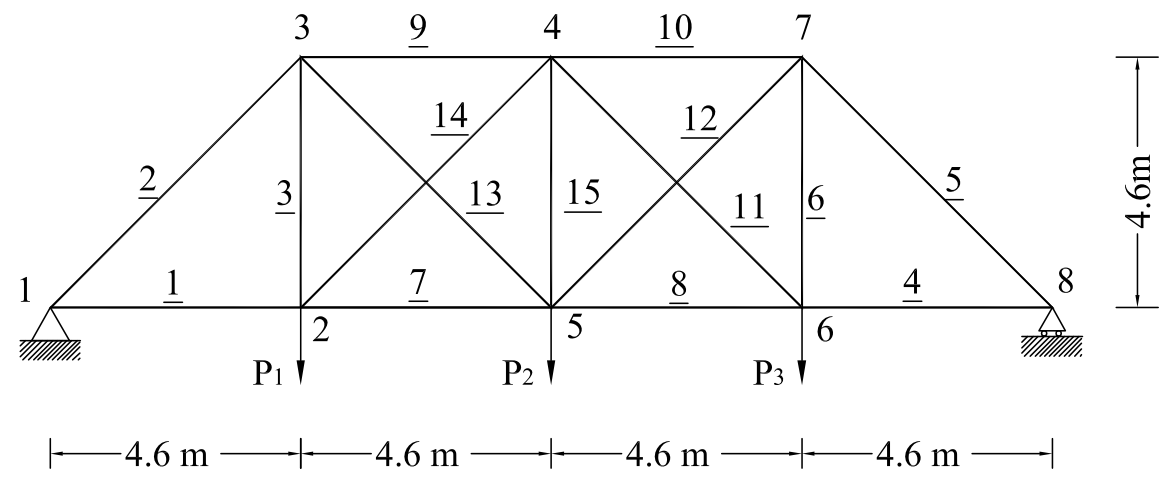

Fig. 3. Truss structure

Table 1. Sample statistics for the basic random variables (truss in Fig. 3)

\begin{tabular}{llll}
\hline Variables & Sample mean & Sample standard deviation & No. of samples \\
\hline A1 $-\mathrm{A} 6\left(\mathrm{~cm}^{2}\right)$ & 10.32 & 0.516 & 30 \\
$\mathrm{~A} 7-\mathrm{A} 15\left(\mathrm{~cm}^{2}\right)$ & 6.45 & 0.323 & 30 \\
$\mathrm{Ln} \mathrm{P}_{1}$ & 3.2122 & 0.071474 & 20 \\
$\mathrm{Ln} \mathrm{P}_{2}$ & 3.9982 & 0.071474 & 20 \\
Ln $P_{3}$ & 3.2122 & 0.071474 & 20 \\
\hline
\end{tabular}

MPa. Interval linear elastic analyses are performed. The element stress is calculated using conventional interval methods (stresses are calculated from interval displacements) as well as the improved algorithm outlined in this paper. The cross-sectional areas for the 15 members and the three loads are identified as the basic random variables. All the 18 random variables are assumed to be mutually statistically independent. Assume that based on experience, the cross-sectional areas can be modeled by normal distributions, and the loads modeled by lognormal distributions. Suppose the statistics for the random variables were estimated from limited samples of data. Table 1 gives the available sample statistics for the cross-sectional areas and the logarithm of the loads ( $\operatorname{Ln} \mathrm{P})$. The Youngs modulus is assumed deterministic (200 GPa).

From the sample size, one can calculate confidence bounds on the mean of the random variables. We will use these bounds to construct the bounding functions defining the probability bounds for the random variables. Two cases are considered (1) the uncertain means for the (logarithm of) loads only and (2) uncertain cross-sectional area as well as loading. The interval Monte Carlo method is used to obtain probability bounds information on the variables associated with the limit states as well as interval estimates for the failure probability. All results are calculated using 10,000 realizations.

Fig. 4 presents the probability bounds for the central deflection of the truss subject to uncertain loading. Using a limit state for deflection of $2.4 \mathrm{~cm}$, the 
Table 2. Probability bounds for the basic random variables (truss in Fig. 3)

\begin{tabular}{lll}
\hline Variables & $\begin{array}{l}\text { Population means 90\% } \\
\text { confidence interval bounds }\end{array}$ & $\begin{array}{l}\text { Population standard } \\
\text { deviation }\end{array}$ \\
\hline A1 $-\mathrm{A} 6\left(\mathrm{~cm}^{2}\right)$ & {$[10.160,10.480]$} & 0.516 \\
$\mathrm{~A} 7-\mathrm{A} 15\left(\mathrm{~cm}^{2}\right)$ & {$[6.3498,6.5502]$} & 0.323 \\
$\mathrm{Ln} \mathrm{P}_{1}$ & {$[3.1846,3.2398]$} & 0.071474 \\
$\mathrm{Ln} \mathrm{P}_{2}$ & {$[3.9706,4.0259]$} & 0.071474 \\
Ln $\mathrm{P}_{3}$ & {$[3.1846,3.2398]$} & 0.071474 \\
\hline
\end{tabular}

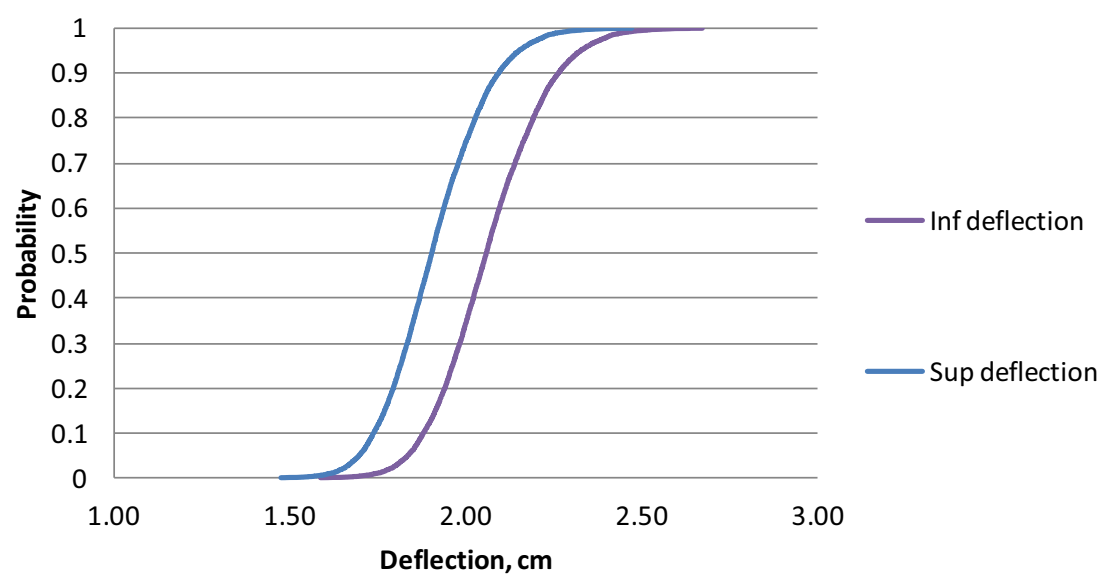

Fig. 4. Probability bounds for central deflection, uncertain load

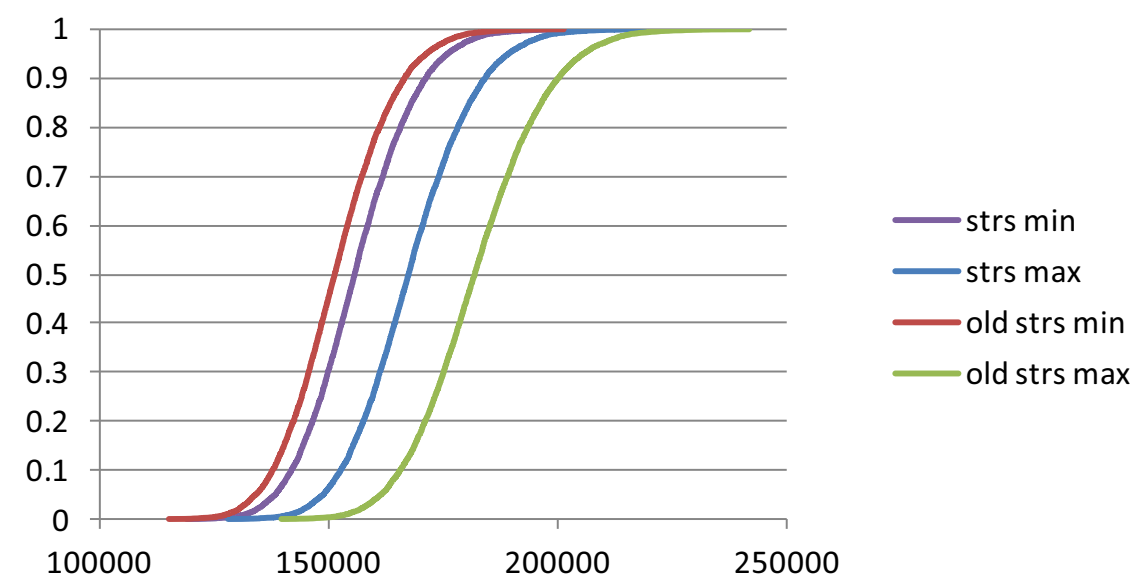

Fig. 5. Probability bounds of maximum absolute stress in the structure, uncertain load 


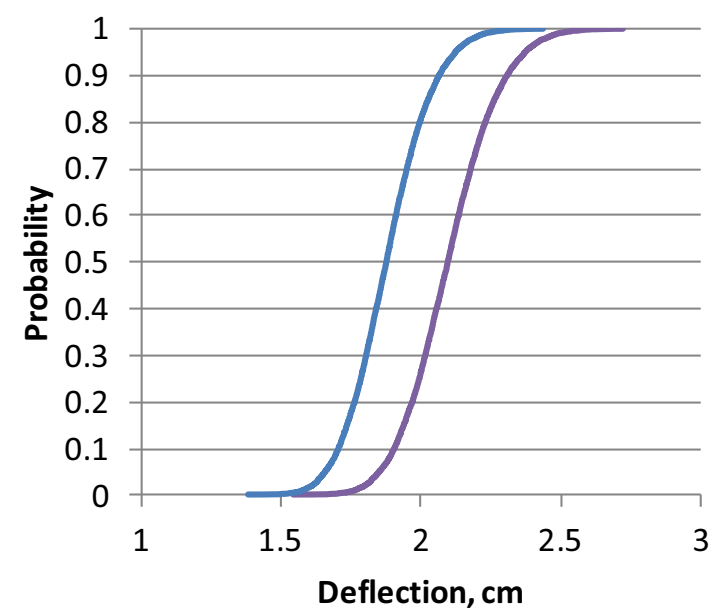

Inf deflection

Sup deflection

Fig. 6. Probability bounds of central deflection for uncertain loads and element crosssectional areas

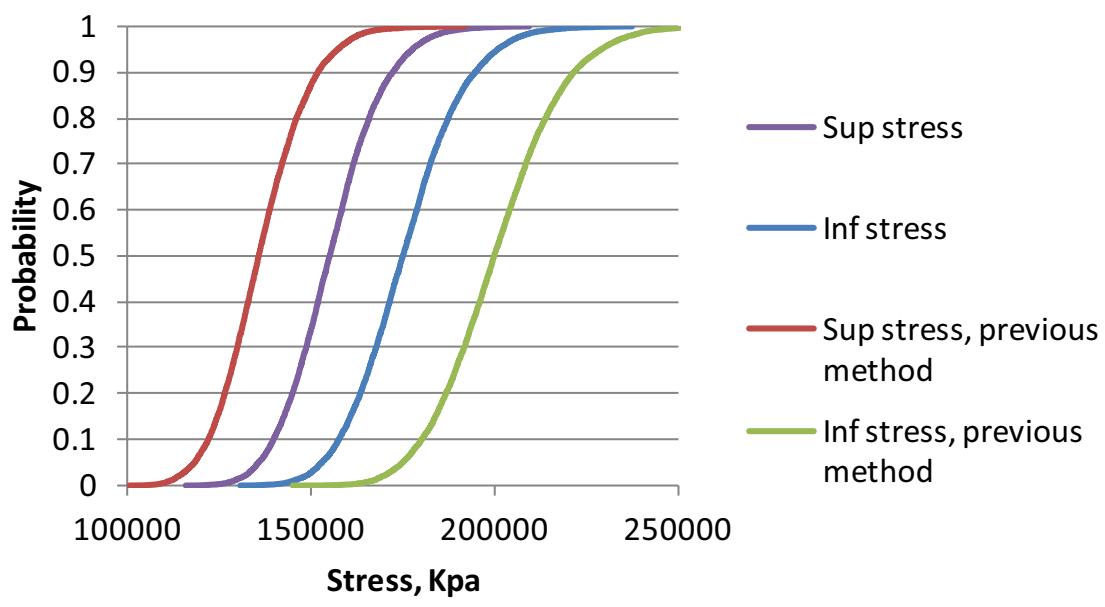

Fig. 7. Probability bounds of maximum absolute stress in the structure, uncertain load and element cross-sectional area

bounds on the probability of survival is given by the intersection of a vertical line at $2.4 \mathrm{~cm}$ and the probability bounds. The failure probability bounds are then calculated from the survival bounds and are $[1.34,1.93] \%$. Fig. 5 presents the probability bounds results for the maximum absolute value of the stress the structure. Two different bounds are presented; the wider bounds are calculated using standard interval methods while the narrower bounds result from the current method. The calculated failure probabilities for the strength-based limit state are: $[0.01,0.76] \%$ using the current method and $[<0.01,9.95] \%$ using the 
previous method. While both results bound the failure probability, the previous method significantly overestimates the bounding values.

Fig. 6 presents the probability bounds for the central deflection of the truss subject to uncertain load and element cross-sectional areas. Using the same 2.4 $\mathrm{cm}$ limit state, the bounds on the probability of failure increase to $[0.11,5.39] \%$. It should be expected that increasing the uncertainty associated with the analysis will increase the width of the bounds on failure probability. Fig. 7 presents the probability bounds results for the maximum absolute value of the stress in the structure. Again, two different bounds are presented: the new method of this work and previous interval method. Using the same limit state, the failure probabilities bound are: $[0.04,3.71] \%$ using the new method and $[<0.01,48.88] \%$ using the previous method. The overestimation using previous method of calculations of element stress clearly renders the previous method useless in engineering design.

\section{Conclusion}

An interval Monte Carlo finite element method with improved calculation of the bounds of secondary quantities is presented. Using element stress as an example, the improved sharpness of the bounds is illustrated. This work resolves the issue of engineering design with limit states calculated from secondary quantities (stress) that existed in previous interval Monte Carlo finite element analyses.

\section{References}

1. Melchers, R.E.: Structural Reliability Analysis and Prediction, 2nd edn. John Wiley \& Sons, West Sussex (1999)

2. Ferson, S., Ginzburg, L.R.: Different Methods Are Needed to Propagate Ignorance and Variability. Reliab. Engng. Syst. Saf. 54, 133-144 (1996)

3. Sentz, K., Ferson, S.: Combination of Evidence in Dempster-Shafer Theory. Technical Report SAND2002-0835, Sandia National Laboratories (2002)

4. Walley, P.: Statistical Reasoning with Imprecise Probabilities. Chapman and Hall, London (1991)

5. Zadeh, L.A.: Fuzzy Sets as a Basis for a Theory of Possibility. Fuzzy Sets and Systems 1, 3-28 (1978)

6. Dempster, A.P.: Upper and Lower Probabilities Induced by a Multi-Valued Mapping. Ann. Mat. Stat. 38, 325-339 (1967)

7. Shafer, G.: A Mathematical Theory of Evidence. Princeton University Press, Princeton (1976)

8. Kendall, D.G.: Foundations of a Theory of Random Sets. In: Harding, E., Kendall, D. (eds.) Stochastic Geometry, New York, pp. 322-376 (1974)

9. Berleant, D.: Automatically Verified Reasoning with Both Intervals and Probability Density Functions. Interval Computations 2, 48-70 (1993)

10. Ferson, S., Kreinovich, V., Ginzburg, L., Myers, D.S., Sentz, K.: Constructing Probability Boxes and Dempster-Shafer Structures. Technical Report SAND20024015, Sandia National Laboratories (2003) 
11. Ben-Haim, Y., Elishakoff, I.: Convex Models of Uncertainty in Applied Mechanics. Elsevier Science, Amsterdam (1990)

12. Moore, R.E.: Interval Analysis. Prentice Hall, Englewood Cliffs (1966)

13. Lodwick, W.A., Jamison, K.D.: Special Issue: Interface between Fuzzy Set Theory and Interval Analysis. Fuzzy Sets and Systems 135, 1-3 (2002)

14. Muhanna, R.L., Mullen, R.L.: Development of Interval Based Methods for Fuzziness in Continuum Mechanics. In: Proc. ISUMA-NAFIPS 1995, pp. 23-45 (1995)

15. Zhang, H., Mullen, R.L., Muhanna, R.L.: Interval Monte Carlo Methods for Structural Reliability. Structural Safety 32, 183-190 (2010)

16. Yager, R.R.: Arithmetic and Other Operations on Dempster-Shafer Structures. International Journal of Man-Machine Studies 25, 357-366 (1986)

17. Williamson, R., Downs, T.: Probabilistic Arithmetic I: Numerical Methods for Calculating Convolutions and Dependency Bounds. International Journal of Approximate Reasoning 4, 89-158 (1990)

18. Berleant, D., Goodman-Strauss, C.: Bounding the Results of Arithmetic Operations on Random Variables of Unknown Dependency Using Intervals. Reliable Computing 4(2), 147-165 (1998)

19. Tonon, F., Bernardini, A., Mammino, A.: Reliability Analysis of Rock Mass Response by Means of Random Set Theory. Reliab. Engng. Syst. Saf. 70, 263-282 (2000)

20. Tonon, F.: A Search Algorithm for Calculating Validated Reliability Bounds. In: Proceedings of NSF Workshop on Reliable Engineering Computing (2004), http://savannah.gatech.edu/workshop/rec04/

21. Joslyn, C., Kreinovich, V.: Convergence Properties of an Interval Probabilistic Approach to System Reliability Estimation. Report No. LA-UR-02-6261, Los Alamos National Laboratory, Los Alamos, NM (2002)

22. Ferson, S., Ginzburg, L.: Hybrid Arithmetic. In: IEEE Proceedings of ISUMA - NAFIPS 1995 The Third International Symposium on Uncertainty Modeling and Analysis and Annual Conference of the North American Fuzzy Information Processing Society, pp. 619-623 (1995)

23. Koyluoglu, U., Cakmak, S., Ahmet, N., Soren, R.K.: Interval Algebra to Deal with Pattern Loading and Structural Uncertainty. Journal of Engineering Mechanics 121(11), 1149-1157 (1995)

24. Muhanna, R.L., Mullen, R.L.: Development of Interval Based Methods for Fuzziness in Continuum Mechanics. In: IEEE Proceedings of ISUMA - NAFIPS 1995 The Third International Symposium on Uncertainty Modeling and Analysis and Annual Conference of the North American Fuzzy Information Processing Society, pp. 145-150 (1995)

25. Rao, S.S., Sawyer, P.: Fuzzy Finite Element Approach for Analysis of Imprecisely Defined Systems. American Institute of Aeronautics and Astronautics Journal 33(12), 2364-2370 (1995)

26. Nakagiri, S., Yoshikawa, N.: Finite Element Interval Estimation by Convex Model. In: Proceedings of 7th ASCE EMD/STD Joint Specialty Conference on Probabilistic Mechanics and Structural Reliability, pp. 278-281. WPI, Worcester (1996)

27. Rao, S.S., Berke, L.: Analysis of Uncertain Structural Systems using Interval Analysis. American Institute of Aeronautics and Astronautics Journal 35(4), 727-735 (1997)

28. Rao, S.S., Chen, L.: Numerical Solution of Fuzzy Linear Equations in Engineering Analysis. International Journal of Numerical Methods in Engineering 43, 391-408 (1998) 
29. Muhanna, R.L., Mullen, R.L.: Uncertainty in Mechanics Problems-Interval-based Approach. Journal of Engineering Mechanics 127(6), 557-566 (2001)

30. Zhang, H.: Nondeterministic Linear Static Finite Element Analysis: An Interval Approach. PhD Dissertation, School of Civil and Environmental Engineering, Georgia Institute of Technology, Atlanta, GA (2005)

31. Rao, M.V.R., Mullen, R.L., Muhanna, R.L.: A New Interval Finite Element Formulation with the Same Accuracy in Primary and Derived Variables. Int. J. Reliability and Safety 5(3/4) (2011)

32. Bathe, K.: Finite Element Procedures. Prentice Hall, Englewood Cliffs (1996)

33. Gallagher, R.H.: Finite Element Analysis Fundamentals. Prentice Hall, Englewood Cliffs (1975)

34. Neumaier, A., Pownuk, A.: Linear Systems with Large Uncertainties, with Applications to Truss Structures. Reliable Computing 13(2), 149-172 (2007) 


\section{Discussion}

Speaker: Rafi Muhanna

Richard Hanson: Does the use of interval arithmetic in finte element methods scale to the size of the structure show in your slide (large!)?

Rafi Muhanna: Yes. We have tested that and the results are reported in following paper: Muhanna, R., Mullen, R., and Zhang, H., "Interval Finite Element as a Basis for Generalized Models of Uncertainty in Engineering Mechanics," Reliable Computing Journal, Springer Netherlands, Vol. 13, No. 2, pp. 173-194, April 2007, and it has been found that the computer time scaled cubically to the number of interval variables which is similar to the conventional finite elements.

William Kahan: This talk illustrates that the use of interval arithmetic to get bounds not grossly excessive requires close analysis and exploitation of a problem's properties. Here the ideas that work on static analysis by finite elements of elastic structures also works on elliptic partial differential equations. But the methods presented here do not always succeed on dynamical systems, nor on solutions of nonlinear systems of equations with interval coefficients. The right person to ask about interval arithmetic's successes is Professor Ulrich Kulisch in attendence here.

Ulrich Kulisch: Yes, for dynamical systems double precision interval and floating-point arithmetic are not very successful. Here long interval arithmetic is the appropriate tool. For the logistic equation $x_{n+1}:=3.75 x_{n}\left(1-x_{n}\right), n \geq 0$, and the initial value $x_{0}=0.5$ double precision floating-point and interval arithmetic totally fail (no correct digit) after 30 iterations. Long interval arithmetic still computes correct digits of a guaranteed enclosure after 2790 iterations.

Rafi Muhanna: Note also that nonlinear structural problems have been successfuly solved and reported in: ICASP'11: Applications of Statistics and Probability in Civil Engineering, Faber, Köhler and Nishijima (eds.), Taylor \& Francis Group, London (2011), with the title: "Interval finite elements for nonlinear material problems." Linear dynamic problems are addressed in: Modares, M., Mullen, R., L. and Muhanna, R. L. "Natural Frequency of a Structure with Bounded Uncertainty," Journal of Engineering Mechanics, ASCE, Vol. 132, No. 12, pp. 1363-1371, 2006.

Ronald Boisvert: Have you written your own interval arithmetic software infrastructure, or are you using tools developed elsewhere?

Rafi Muhanna: The results in this presentation are calculated using MATLAB with the INTLAB toolbox. 
Jeffrey Fong: Log normal or normal distributions are not suitable for the modeling of ultimate tensile strength data because they do not predict a minimum strength. A 3-parameter Weibull distribution with a positive location parameter is a better model for predicting a minimum strength. Do you have experience using Weibull in interval finite elements?

Rafi Muhanna: In this work we did not model the material strength. The authors have studied probability bounds using a 3-parameter Weibull distribution. The results are not yet published. 